\title{
Novel C-Geranyl 7-Hydroxycoumarins from the Aerial Parts of Eriostemon tomentellus
}

\author{
Mohammad A. Rashid ${ }^{\mathrm{a}}$, James A. Armstrong ${ }^{\mathrm{b}}$, Alexander I. Gray ${ }^{\mathrm{a}}$, and Peter G. Waterman ${ }^{\mathrm{a}, *}$ \\ ${ }^{a}$ Phytochemistry Research Laboratories, Department of Pharmacy, \\ University of Strathclyde, Glasgow G 11 XW, Scotland, U.K. \\ b Western Australian Herbarium, Department of Conservation and Land Management, \\ PO Box 104, Como, Western Australia 6152
}

Z. Naturforsch. 47 b, 284-287 (1992); received Juni 26, 1991

Rutaceae, Eriostemon, 6- and 8-Geranyl-7-hydroxycoumarins, Amide, Tembamide

From the aerial parts of Eriostemon tomentellus five coumarins have been isolated. These have been characterized as ostruthin (6-geranyl-7-hydroxycoumarin (1)) and the novel compounds, 8-geranyl-7-hydroxycoumarin (2), (E)-6-(7-hydroxy-3,7-dimethylocta-2,5-dienyl)7-hydroxycoumarin (3), (E)-6-(7-hydroperoxy-3,7-dimethylocta-2,5-dienyl)-7-hydroxycoumarin (4), and 6-(6-hydroxy-3,7-dimethylocta-2,7-dienyl)-7-hydroxycoumarin (5). In addition tembamide (2-\{4-methoxyphenyl $\}-2$-hydroxy-N-benzoylethylamine (6) was isolated and the ${ }^{13} \mathrm{C}$ NMR spectrum reported for the first time.

\section{Introduction}

Eriostemon tomentellus Diels is a minutely tomentose undershrub found widely in the southwestern part of Western Australia [1]. Previous investigations have revealed the presence of the furoquinoline alkaloids skimmianine and maculosidine, the dihydrocinnamic acid derivative eriostemoic acid, and the coumarin ostruthin (1) [2]. We have recently been able to obtain a further sample and in this paper we wish to report the isolation and identification of a number of novel coumarins.

\section{Results and Discussion}

Sequential Soxhlet extraction of the ground aerial parts with petroleum ether (b.p. $60-80^{\circ} \mathrm{C}$ ), ethyl acetate and methanol followed by column chromatography of each extract over silica gel and then, in some cases, preparative TLC gave: (a) from the petroleum ether extract eriostemoic acid, ostruthin (1), maculosidine and one novel coumarin (b) from the ethyl acetate extract tembamide (6) and three further novel coumarins, and (c) from the methanol extract the flavanone glycoside hesperidin.

The novel coumarin from the petroleum ether extract showed $\mathrm{M}^{+} 298$, resolving by high resolution electron impact MS for $\mathrm{C}_{19} \mathrm{H}_{22} \mathrm{O}_{3}$. The ${ }^{1} \mathrm{H}$

\footnotetext{
* Reprint requests to Prof. P. G. Waterman.

Verlag der Zeitschrift für Naturforschung, D-W-7400 Tübingen $0932-0776 / 92 / 0200-0284 / \$ 01.00 / 0$
}

NMR spectrum (Table I) revealed signals for AB-coupling between $\mathrm{H}-3$ and $\mathrm{H}-4$ and between $\mathrm{H}-5$ and $\mathrm{H}-6$. Other resonances showed couplings identical to those observed for the geranyl sidechain in $\mathbf{1}$ (Table I). The presence of the geranyl side-chain was further confirmed by the presence of a fragment $m / z 123\left[\mathrm{C}_{9} \mathrm{H}_{15}\right]^{+}$for fission of the side-chain between $\mathrm{C}-1^{\prime}$ and $\mathrm{C}-2^{\prime}$. On this basis the compound must be the isomer of ostruthin with structure 2 .

The most polar of the coumarins from the ethyl acetate extract gave a highest fragment in the electron impact MS at $m / z 296\left[\mathrm{C}_{19} \mathrm{H}_{20} \mathrm{O}_{3}\right]^{+}$but fast atom bombardment MS revealed $\mathrm{M}^{+}+1$ at $\mathrm{m} / \mathrm{z}$ 315 indicating an empirical formula $\mathrm{C}_{19} \mathrm{H}_{22} \mathrm{O}_{4}$, one oxygen more than $\mathbf{1}$ and $\mathbf{2}$. The ${ }^{1} \mathrm{H}$ NMR spectrum (Table I) revealed signals for $\mathrm{H}-3, \mathrm{H}-4, \mathrm{H}-5$ and $\mathrm{H}-8$ of the coumarin nucleus, requiring a 6-geranyl-7-hydroxycoumarin structure. A feature of the ${ }^{1} \mathrm{H}$ NMR spectrum was the presence of three olefinic protons attributable to the $\mathrm{C}_{10}$ side chain; one of these was typical for $\mathrm{H}-2^{\prime}$ while the others showed coupling to each other of $15.4 \mathrm{~Hz}$, indicative of a trans relationship with one showing further coupling to a methylene group. Another feature of the ${ }^{1} \mathrm{H}$ NMR spectrum was the shielding of two methyl resonances to $\delta 1.34$, typical for geminal methyls attached to a carbon also carrying an oxygen substituent.

Placement of the methylene at C-4' rather than C-6' was established by the observation of a nuclear Overhauser interaction between $\mathrm{H}-2^{\prime}$ and the 
Table I. 'H NMR spectra for coumarins 1-5.

\begin{tabular}{|c|c|c|c|c|c|}
\hline & 1 & 2 & 3 & 4 & 5 \\
\hline $\mathrm{H}-3$ & $\begin{array}{l}6.24(1 \mathrm{H}, \mathrm{d} \\
J=9.5 \mathrm{~Hz})\end{array}$ & $\begin{array}{l}6.25(1 \mathrm{H}, \mathrm{d}, \\
J=9.5 \mathrm{~Hz})\end{array}$ & $\begin{array}{l}6.21(1 \mathrm{H}, \mathrm{d}, \\
J=9.4 \mathrm{~Hz})\end{array}$ & $\begin{array}{l}6.24(1 \mathrm{H}, \mathrm{d}, \\
J=9.5 \mathrm{~Hz})\end{array}$ & $\begin{array}{l}6.26(1 \mathrm{H}, \mathrm{d}, \\
J=9.3 \mathrm{~Hz})\end{array}$ \\
\hline $\mathrm{H}-4$ & $\begin{array}{l}7.65(1 \mathrm{H}, \mathrm{d}, \\
J=9.5 \mathrm{~Hz})\end{array}$ & $\begin{array}{l}7.64(1 \mathrm{H}, \mathrm{d}, \\
J=9.5 \mathrm{~Hz})\end{array}$ & $\begin{array}{l}7.62(1 \mathrm{H}, \mathrm{d}, \\
J=9.4 \mathrm{~Hz})\end{array}$ & $\begin{array}{l}7.65(1 \mathrm{H}, \mathrm{d}, \\
J=9.5 \mathrm{~Hz})\end{array}$ & $\begin{array}{l}7.67(1 \mathrm{H}, \mathrm{d}, \\
J=9.3 \mathrm{~Hz})\end{array}$ \\
\hline $\mathrm{H}-5$ & $7.20 \mathrm{~s}$ & $\begin{array}{l}7.25(1 \mathrm{H}, \mathrm{d}, \\
J=8.4 \mathrm{~Hz})\end{array}$ & $7.17 \mathrm{~s}$ & $7.19 \mathrm{~s}$ & $7.38 \mathrm{~s}$ \\
\hline H-6 & & $\begin{array}{l}6.81(1 \mathrm{H}, \mathrm{d}, \\
J=8.4 \mathrm{~Hz})\end{array}$ & & & \\
\hline $\mathrm{H}-8$ & $7.01 \mathrm{~s}$ & & $6.95 \mathrm{~s}$ & $7.00 \mathrm{~s}$ & $6.97 \mathrm{~s}$ \\
\hline $\mathrm{H}-1^{\prime}$ & $\begin{array}{l}3.40(2 \mathrm{H}, \mathrm{d}, \\
J=7.2 \mathrm{~Hz})\end{array}$ & $\begin{array}{l}3.66(2 \mathrm{H}, \mathrm{d}, \\
J=6.9 \mathrm{~Hz})\end{array}$ & $\begin{array}{l}3.37(2 \mathrm{H}, \mathrm{d}, \\
J=7.4 \mathrm{~Hz})\end{array}$ & $\begin{array}{l}3.39(2 \mathrm{H}, \mathrm{d}, \\
J=7.2 \mathrm{~Hz})\end{array}$ & $\begin{array}{l}3.65(2 \mathrm{H}, \mathrm{d}, \\
J=7.4 \mathrm{~Hz})\end{array}$ \\
\hline $\mathrm{H}-2^{\prime}$ & $\begin{array}{l}5.33(1 \mathrm{H}, \mathrm{tq}, \\
J=7.2,1.2 \mathrm{~Hz})\end{array}$ & $\begin{array}{l}5.29(1 \mathrm{H}, \mathrm{tq}, \\
J=6.9,1.1 \mathrm{~Hz})\end{array}$ & $\begin{array}{l}5.34(1 \mathrm{H}, \text { br.t, } \\
J=7.4 \mathrm{~Hz})\end{array}$ & $\begin{array}{l}5.38(1 \mathrm{H}, \text { br.t } \\
J=7.2 \mathrm{~Hz})\end{array}$ & $\begin{array}{l}5.72(1 \mathrm{H}, \mathrm{tq}, \\
J=7.4,1.3 \mathrm{~Hz})\end{array}$ \\
\hline $\mathrm{CH}_{3}-3^{\prime}$ & $\begin{array}{l}1.74(3 \mathrm{H}, \mathrm{d} \\
J=1.2 \mathrm{~Hz})\end{array}$ & $\begin{array}{l}1.86(3 \mathrm{H}, \mathrm{d} \\
J=1.1 \mathrm{~Hz})\end{array}$ & $1.71(3 \mathrm{H}, \mathrm{br} . \mathrm{s})$ & 1.74 (3 H, br.s) & $\begin{array}{l}1.82(3 \mathrm{H}, \mathrm{d} \\
J=1.3 \mathrm{~Hz})\end{array}$ \\
\hline $\mathrm{H}-4^{\prime}$ & $2.10(2 \mathrm{H}, \mathrm{m})$ & $2.10(2 \mathrm{H}, \mathrm{m})$ & $\begin{array}{l}2.76(2 \mathrm{H}, \mathrm{br} . \mathrm{d} \\
J=5.6 \mathrm{~Hz})\end{array}$ & $\begin{array}{l}2.80(2 \mathrm{H}, \mathrm{br} . \mathrm{d}, \\
J=6.6 \mathrm{~Hz})\end{array}$ & $2.38(2 \mathrm{H}, \mathrm{m})$ \\
\hline $\mathrm{H}-5^{\prime}$ & $2.10(2 \mathrm{H}, \mathrm{m})$ & $2.10(2 \mathrm{H}, \mathrm{m})$ & $\begin{array}{l}5.65(1 \mathrm{H}, \mathrm{dt} \\
J=15.4,5.6 \mathrm{~Hz})\end{array}$ & $\begin{array}{l}5.72(2 \mathrm{H}, \mathrm{dt} \\
J=15.7,6.6 \mathrm{~Hz})\end{array}$ & $1.95(2 \mathrm{H}, \mathrm{m})$ \\
\hline H- $6^{\prime}$ & $\begin{array}{l}5.09(1 \mathrm{H}, \text { br.t, } \\
J=6.8 \mathrm{~Hz})\end{array}$ & $\begin{array}{l}5.05(1 \mathrm{H}, \text { br.t } \\
J=6.7 \mathrm{~Hz})\end{array}$ & $\begin{array}{l}5.67(1 \mathrm{H}, \mathrm{d} \\
J=15.4 \mathrm{~Hz})\end{array}$ & $\begin{array}{l}5.61(1 \mathrm{H}, \mathrm{d} \\
J=15.7 \mathrm{~Hz})\end{array}$ & $\begin{array}{l}4.37(1 \mathrm{H}, \mathrm{t} \\
J=6.7 \mathrm{~Hz})\end{array}$ \\
\hline $\mathrm{CH}_{3}-7^{\prime}$ & $1.60(3 \mathrm{H}$, br.s $)$ & $1.60(3 \mathrm{H}$, br.s $)$ & $1.34(3 \mathrm{H}, \mathrm{s})$ & $1.35(3 \mathrm{H}, \mathrm{s})$ & $1.86(3 \mathrm{H}$, br.s $)$ \\
\hline $\mathrm{H}-8^{\prime}$ & $1.69(3 \mathrm{H}, \mathrm{br} . \mathrm{s})$ & $1.68(3 \mathrm{H}$, br.s $)$ & $1.34(3 \mathrm{H}, \mathrm{s})$ & $1.35(3 \mathrm{H}, \mathrm{s})$ & $\begin{array}{l}4.93(1 \mathrm{H}, \mathrm{m}) \\
5.22(1 \mathrm{H}, \mathrm{m})\end{array}$ \\
\hline OOH-7' & & & & 6.90 (1 H, br.s) & \\
\hline
\end{tabular}

1 was run at $250 \mathrm{MHz}$ in $\mathrm{CDCl}_{3}, \mathbf{2 - 4}$ at $400 \mathrm{MHz}$ in $\mathrm{CDCl}_{3}$ and 5 at $400 \mathrm{MHz}$ in $\mathrm{C}_{5} \mathrm{D}_{5} \mathrm{~N}$.

H-4' methylene protons. The nOe also confirmed a cis relationship between $\mathrm{H}-\mathrm{I}^{\prime}$ and the $3^{\prime}$-methyl allowing assignment of structure 3 . Finally a heteronuclear multiple bond coupling (HMBC) study [3] was used to assign the ${ }^{13} \mathrm{C}$ NMR chemical shifts (Table II).

The least polar coumarin from the ethyl acetate extract also failed to show an $\mathrm{M}^{+}$in the electron impact MS but fast atom bombardment MS showed a pseudomolecular ion $\left[\mathrm{M}^{+}+1\right]$ at $\mathrm{m} / \mathrm{z}$ 331 , indicative of an empirical formula $\mathrm{C}_{19} \mathrm{H}_{22} \mathrm{O}_{5}$. Significant fragments in the electron impact $\mathrm{MS}$ could be allocated to the loss of $\mathrm{OOH}^{-}$and $\mathrm{H}_{2} \mathrm{O}_{2}$, suggesting the presence of a hydroperoxy group. The ${ }^{1} \mathrm{H}$ NMR spectrum (Table I) and ${ }^{13} \mathrm{C}$ NMR spectrum (Table II) were closely comparable to those of $\mathbf{3}$ for the coumarin nucleus and for $\mathrm{C}-\mathrm{1}^{\prime}$ to C-4' of the side chain but the carbon spectrum showed strong deshielding for $\mathrm{C}-5^{\prime}$ and $\mathrm{C}-7^{\prime}$ and shielding for $\mathrm{C}-6^{\prime}, \mathrm{C}-8^{\prime}$ and the $\mathrm{C}-7^{\prime}$ methyl. These
Table II. ${ }^{13} \mathrm{C}$ NMR spectra for 3-5.

\begin{tabular}{lrrr}
\hline Carbon & 3 & 4 & 5 \\
\hline 2 & $162.2 \mathrm{~s}$ & $162.4 \mathrm{~s}$ & $162.2 \mathrm{~s}$ \\
3 & $112.2 \mathrm{~d}$ & $112.2 \mathrm{~d}$ & $112.3 \mathrm{~d}$ \\
4 & $144.1 \mathrm{~d}$ & $144.2 \mathrm{~d}$ & $144.1 \mathrm{~d}$ \\
5 & $128.3 \mathrm{~d}$ & $128.2 \mathrm{~d}$ & $128.4 \mathrm{~d}$ \\
6 & $125.6 \mathrm{~s}$ & $125.6 \mathrm{~s}$ & $125.6 \mathrm{~s}$ \\
7 & $158.5 \mathrm{~s}$ & $158.4 \mathrm{~s}$ & $158.5 \mathrm{~s}$ \\
8 & $103.1 \mathrm{~d}$ & $103.1 \mathrm{~d}$ & $103.1 \mathrm{~d}$ \\
9 & $154.1 \mathrm{~s}$ & $154.1 \mathrm{~s}$ & $154.1 \mathrm{~s}$ \\
10 & $112.2 \mathrm{~s}$ & $112.2 \mathrm{~s}$ & $112.2 \mathrm{~s}$ \\
$1^{\prime}$ & $28.4 \mathrm{t}$ & $28.4 \mathrm{t}$ & $28.6 \mathrm{t}$ \\
$2^{\prime}$ & $122.0 \mathrm{~d}$ & $122.2 \mathrm{~d}$ & $121.7 \mathrm{~d}$ \\
$3^{\prime}$ & $137.0 \mathrm{~s}$ & $136.7 \mathrm{~s}$ & $137.9 \mathrm{~s}$ \\
$3^{\prime}-\mathrm{CH}_{3}$ & $16.3 \mathrm{q}$ & $16.3 \mathrm{q}$ & $16.2 \mathrm{q}$ \\
$4^{\prime}$ & $42.3 \mathrm{t}$ & $42.5 \mathrm{t}$ & $* 32.9 \mathrm{t}$ \\
$5^{\prime}$ & $124.8 \mathrm{~d}$ & $129.4 \mathrm{~d}$ & $* 35.8 \mathrm{t}$ \\
$6^{\prime}$ & $139.6 \mathrm{~d}$ & $135.1 \mathrm{~d}$ & $75.9 \mathrm{~d}$ \\
$7^{\prime}$ & $71.1 \mathrm{~s}$ & $82.2 \mathrm{~s}$ & $147.1 \mathrm{~s}$ \\
$7^{\prime}-\mathrm{CH}_{3}$ & $29.8 \mathrm{q}$ & $24.2 \mathrm{q}$ & $17.7 \mathrm{q}$ \\
$8^{\prime}$ & $29.8 \mathrm{q}$ & $24.2 \mathrm{q}$ & $111.3 \mathrm{t}$ \\
\hline
\end{tabular}

* Assignments interchangeable; spectra run at 100.6 $\mathrm{MHz}$ in $\mathrm{CDCl}_{3}$. 
changes are in agreement with those observed in conversion of an isopropanol terminal group to the corresponding peroxide and permit assignment of structure 4 [4]. Again an HMBC study allowed unambiguous assignment of all carbon resonances.

The final coumarin showed a comparable coumarin nucleus and $\mathrm{C}-\mathbf{1}^{\prime}$ to $\mathrm{C}-3^{\prime}$ side chain to 3 and 4. The electron impact MS showed $\mathrm{M}^{+} 314$, solving for $\mathrm{C}_{19} \mathrm{H}_{22} \mathrm{O}_{4}$, identical to 3 . Differences from 3 observed in both ${ }^{1} \mathrm{H}$ (Table I) and ${ }^{13} \mathrm{C}$ (Table II) NMR spectra indicated the presence of a terminal isopropenyl moiety and a secondary alcohol. Placement of the hydroxyl at C-6' was established from a NOESY study which showed a strong interaction between the $\delta 1.86$ methyl of the isopropenyl group and the oxymethine proton at $\delta 4.37$. On this basis the coumarin can be assigned structure 5 , the stereochemistry of the 6 '-hydroxyl being unknown.

Tembamide (6) was also isolated from the ethyl acetate extract. While this amide has been found quite widely in the Rutaceae [5] this is the first report of its occurrence in Eriostemon. The opportunity was taken to record, for the first time, the ${ }^{13} \mathrm{C}$ NMR spectrum, assignments again being made unambiguously through application of the $\mathrm{HMBC}$ technique.<smiles>CC(C)=CCCC(C)=CCc1cc2ccc(=O)oc2cc1O</smiles>

1

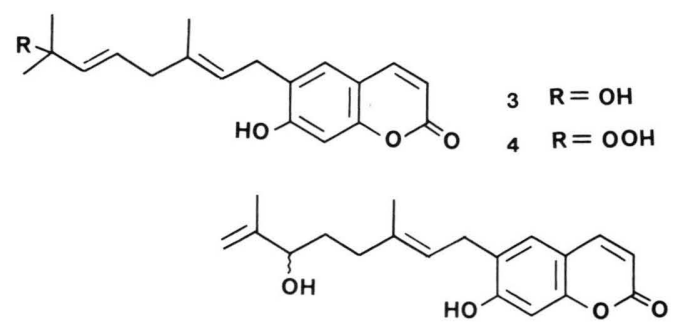

5<smiles>COc1ccc(C(O)CNC(=O)c2ccccc2)cc1</smiles>

\section{Experimental}

\section{Plant material}

Eriostemon tomentellus was collected on September 5, 1989 from Ennuin Station, South Western Australia. A voucher specimen, Perth 01185853 has been deposited at the Western Australian Herbarium.

\section{Isolation of compounds}

The ground aerial parts $(300 \mathrm{~g})$ were extracted successively with petroleum ether (b. p. $60-80^{\circ} \mathrm{C}$ ), EtOAc and $\mathrm{MeOH}$. The petrol extract was concentrated $(15 \mathrm{~g})$ and an aliquot $(5 \mathrm{~g})$ subjected to vacuum liquid chromatography over $\mathrm{Si}$ gel, eluting with petrol containing increasing amounts of EtOAc. Elution with $20 \%$ EtOAc, followed by removal of pigments (Sephadex LH 20 column eluting with $\mathrm{CHCl}_{3}$ ) gave eriostemoic acid $(274 \mathrm{mg})$. Further elution with $30 \%$ EtOAc, followed by treatment with Sephadex LH20 and then PTLC (Si gel, solvent toluene: EtOAc 8:2), gave 2 (4 mg) and $\mathbf{1}(215 \mathrm{mg})$. Finally elution with EtOAc gave maculosidine $(5 \mathrm{mg})$.

Concentration of the EtOAc extract gave a solid $(11 \mathrm{~g})$ an aliquot of which $(7 \mathrm{~g})$ was subjected to vacuum liquid chromatography eluting with petrol:EtOAc and then EtOAc: $\mathrm{MeOH}$ mixtures of increasing polarity. Elution with $50 \%$ EtOAc in petrol yielded a mixture which was separated by PTLC (Si gel, solvent $\mathrm{CHCl}_{3}: \mathrm{MeOH} 49: 1$ ) to give $4(16 \mathrm{mg})$ and $5(13 \mathrm{mg})$. Concentration of the $65 \%$ EtOAc in petrol eluant gave crystals of 6 $(13 \mathrm{mg})$. PTLC of the supernatant from which $\mathbf{6}$ had been crystallized (Si gel, toluene: EtOAc $8: 3$ ) yielded $3(6 \mathrm{mg})$.

Concentration of the $\mathrm{MeOH}$ extract yielded small amounts of hesperidin (12 mg).

\section{Eriostemoic acid}

Identical (UV, IR, ${ }^{1} \mathrm{H}$ NMR, MS) with authentic sample [6].

\section{Ostruthin (1)}

Plates from petrol:EtOAc, m.p. $117-119^{\circ} \mathrm{C}$ (Lit. [7] $117-118^{\circ} \mathrm{C}$ ). UV $\lambda_{\max }$ [nm]: $\mathrm{MeOH} 245$, 257, 334. IR $v_{\max }\left[\mathrm{cm}^{-1}\right]: \mathrm{KBr} 3160,1685,1600$, $1570,1440,1390,1270,1235,1150,1130,980,850$, 745, 660. ${ }^{1} \mathrm{H}$ NMR - see Table I. MS (rel. int.): $m / z 298$ (34) (calcd for $\mathrm{C}_{19} \mathrm{H}_{22} \mathrm{O}_{3} 298.1569$, found 298.1559), 229 (35), 175 (70), 123 (68), 69 (100). 


\section{8-Geranyl-7-hydroxycoumarin (2)}

Gum. UV $\lambda_{\max }[\mathrm{nm}]: \mathrm{MeOH} 235,253 \mathrm{sh}, 280 \mathrm{sh}$, 322. IR $v_{\max }\left[\mathrm{cm}^{-1}\right]$ : film $3300,1730,1710,1605$, $1570,1450,1255,1120,830 .{ }^{1} \mathrm{H}$ NMR - see Table I. MS (rel. int.): $m / z 298$ (34) (calcd for $\mathrm{C}_{19} \mathrm{H}_{22} \mathrm{O}_{3} 298.1569$, found 298.1585), 229 (59), 175 (100), 123 (98), 69 (48).

\section{Maculosidine}

Identical (mixed m.p., UV, IR, ${ }^{1} \mathrm{H}$ NMR, MS) with authentic sample [6].

\section{(E)-6-(7-Hydroxy-3,7-dimethylocta-2,5-dienyl)- 7-hydroxycoumarin (3)}

Gum. UV $\lambda_{\max }[\mathrm{nm}]: \mathrm{MeOH} 235,256 \mathrm{sh}, 274 \mathrm{sh}$, $280,320 \mathrm{sh}, 331$. IR $v_{\max }\left[\mathrm{cm}^{-1}\right]$ : film 3315,1710 , $1620,1570,1440,1390,1255,1130,820,750 .{ }^{1} \mathrm{H}$ NMR - see Table I. ${ }^{13} \mathrm{C}$ NMR - see Table II. FAB-MS (rel. int.): $m / z 315.1596\left(\mathrm{C}_{19} \mathrm{H}_{23} \mathrm{O}_{4}-\right.$ $\left.\mathrm{M}^{+}+1\right)$. EI-MS (rel. int.): $m / z 296$ (66), 215 (39), $175(100), 121(47)$.

\section{(E)-6-(7-Hydroperoxy-3,7-dimethylocta- 2,5-dienyl)-7-hydroxycoumarin (4)}

Gum. UV $\lambda_{\max }[\mathrm{nm}]: \mathrm{MeOH} 232,244 \mathrm{sh}, 256 \mathrm{sh}$, 330. IR $v_{\max }\left[\mathrm{cm}^{-1}\right]$ : film $3340,1715,1620,1570$, $1440,1390,1260,1135,810,735 .{ }^{1} \mathrm{H}$ NMR - see Table I. ${ }^{13} \mathrm{C}$ NMR - see Table II. FAB-MS (rel. int.): $m / z 331.1545\left(\mathrm{C}_{19} \mathrm{H}_{23} \mathrm{O}_{5}-\mathrm{M}^{+}+1\right)$. EI-MS (rel. int.): $m / z 312$ (6), 297 (2), 296 (12), 229 (5), 175 (100), 137 (11).

\section{6-(6-Hydroxy-3,7-dimethylocta-2,7-dienyl)-} 7-hydroxycoumarin (5)

Gum. $[\alpha]_{\mathrm{D}}+4^{\circ}\left(c=0.3, \mathrm{CHCl}_{3}\right)$. UV $\lambda_{\max }[\mathrm{nm}]:$ $\mathrm{MeOH} 244,258,332$. IR $v_{\max }\left[\mathrm{cm}^{-1}\right]: \mathrm{KBr} 3385$, $3120,1690,1620,1445,1390,1255,1165,1055$,
880, 815. ${ }^{1} \mathrm{H}$ NMR - see Table I. ${ }^{13} \mathrm{C}$ NMR - see Table II. MS (rel. int.): $m / z 314$ (23) (calcd for $\mathrm{C}_{19} \mathrm{H}_{22} \mathrm{O}_{4}$ 314.1518, found 314.1505), 296 (18), 229 (31), $175(100), 147$ (13).

\section{Tembamide (6)}

Needles from petrol:EtOAc, m.p. $146-148^{\circ} \mathrm{C}$ (Lit. [8] $\left.147-149^{\circ} \mathrm{C}\right) .[\alpha]_{\mathrm{D}}+13^{\circ}\left(c=0.3, \mathrm{CHCl}_{3}\right)$ (Lit. [8] $+23^{\circ}$ ). UV $\lambda_{\max }$ [nm]: $\mathrm{MeOH} 228,275,280$. IR $v_{\max }\left[\mathrm{cm}^{-1}\right]: \mathrm{KBr} 3420,3100,1620,1540,1510$, $1300,1245,1180,1080,1030,830,690 .{ }^{1} \mathrm{H}$ NMR $\left(400 \mathrm{MHz}, \mathrm{CDCl}_{3}\right): \delta 3.20(1 \mathrm{H}, \mathrm{d}, J=3.5 \mathrm{~Hz}$, $\mathrm{OH}), 3.51(1 \mathrm{H}$, ddd, $J=11.6,8.0,3.5 \mathrm{~Hz}, \mathrm{H}-1)$, $3.81(3 \mathrm{H}, \mathrm{s}, \mathrm{OMe}), 3.88(1 \mathrm{H}$, ddd, $J=11.6,8.0$, $4.8 \mathrm{~Hz}, \mathrm{H}-1), 4.90(1 \mathrm{H}, \mathrm{dt}, J=3.5,8.0 \mathrm{~Hz}, \mathrm{H}-2)$, $6.62(1 \mathrm{H}, \mathrm{dd}, J=4.8,3.5 \mathrm{~Hz}, \mathrm{~N}-\mathrm{H}), 6.90(2 \mathrm{H}, \mathrm{dd}$, $\left.J=8.8,2.2 \mathrm{~Hz}, \mathrm{H}-3^{\prime}, \mathrm{H}-5^{\prime}\right), 7.34(2 \mathrm{H}, \mathrm{dd}, J=8.8$, $\left.2.2 \mathrm{~Hz}, \mathrm{H}-2^{\prime}, \mathrm{H}-6^{\prime}\right), 7.44(2 \mathrm{H}, \mathrm{dt}, J=1.5,7.3 \mathrm{~Hz}$, $\left.\mathrm{H}-3^{\prime \prime}, \mathrm{H}-5^{\prime \prime}\right), 7.51\left(1 \mathrm{H}, \mathrm{tt}, J=1.5,7.3 \mathrm{~Hz}, \mathrm{H}-4^{\prime \prime}\right)$, $7.76\left(2 \mathrm{H}, \mathrm{dd}, J=7.3,1.5 \mathrm{~Hz}, \mathrm{H}-2^{\prime \prime}, \mathrm{H}-6^{\prime \prime}\right) .{ }^{13} \mathrm{C}$ NMR (100.6 MHz, $\left.\mathrm{CDCl}_{3}\right): \delta 47.7$ (t, C-1), 55.3 (q, $\mathrm{OMe}), 73.3$ (d, C-2), 114.0 (d, C-3', C-5'), 127.0 (d, C-2", C-6"), 127.1 (d, C-2', C-6'), 128.6 (d, C-3", C-5"), 131.7 (s, C-4"), 133.8 (s, C-1'), 134.1 (s, C-1"), 159.3 (s, C-4'), $168.5(\mathrm{~s}, \mathrm{C}=\mathrm{O}$ ). MS (rel. int.): $m / z 271$ (2) (calcd for $\mathrm{C}_{16} \mathrm{H}_{17} \mathrm{NO}_{3} 271.1209$, found 271.1219), $150(61), 137$ (63), 135 (100), 109 (27), 105 (78).

\section{Hesperidin}

Identical (co-TLC, UV, IR, ${ }^{1} \mathrm{H}$ NMR) with authentic sample [6].

One of us (M.A.R.) thanks the Association of Commonwealth Universities for the award of a scholarship. NMR spectra were run in the Strathclyde University NMR Laboratory.
[1] P. G. Wilson, Nuytsia 1, 1 (1970).

[2] A. M. Duffield and P. R. Jefferies, Aust. J. Chem. 16, 123 (1963).

[3] A. Bax and M. F. Summers, J. Am. Chem. Soc. 108, 2093 (1986).

[4] F. S. El-Feraly, D. A. Benigni, and A. T. McPhail, J. Chem. Soc. Perkin Trans. I 1983, 355.

[5] I. Mester, in P. G. Waterman and M. F. Grundon (eds.): Chemistry and Chemical Taxonomy of the Rutales, p. 31, Academic Press, London (1983).

[6] M. A. Rashid, J. A. Armstrong, A. I. Gray, and P. G. Waterman, Phytochemistry, in press.

[7] G. K. Nikonov, G. Yu Pek, and V. V. Vandyshev, Khim. Prior. Soedin. 1968, 318.

[8] S. M. Albonico, A. M. Kuck, and V. Deulofeu, J. Chem. Soc. C 1967, 1327. 